\title{
Evidence of energy-, recombination-, and photon-limited escape regimes in giant planet $\mathrm{H} / \mathrm{He}$ atmospheres
}

\author{
M. Lampón ${ }^{1}$, M. López-Puertas ${ }^{1}$, S. Czesla ${ }^{2}$, A. Sánchez-López ${ }^{3}$, L. M. Lara ${ }^{1}$, M. Salz², J. Sanz-Forcada ${ }^{4}$, \\ K. Molaverdikhani ${ }^{5,6}$, A. Quirrenbach ${ }^{6}$, E. Pallé ${ }^{7,8}$, J. A. Caballero ${ }^{4}$, Th. Henning ${ }^{5}$, L. Nortmann ${ }^{9}$, P. J. Amado ${ }^{1}$, \\ D. Montes ${ }^{10}$, A. Reiners ${ }^{9}$, and I. Ribas ${ }^{11,12}$ \\ ${ }^{1}$ Instituto de Astrofísica de Andalucía (IAA-CSIC), Glorieta de la Astronomía s/n, 18008 Granada, Spain \\ e-mail: mlampon@iaa.es \\ 2 Hamburger Sternwarte, Universität Hamburg,Gojenbergsweg 112, 21029 Hamburg, Germany \\ 3 Leiden Observatory, Leiden University, Postbus 9513, 2300 RA Leiden, The Netherlands \\ ${ }^{4}$ Centro de Astrobiología (CSIC-INTA), ESAC, Camino bajo del castillo s/n, 28692, Villanueva de la Cañada Madrid, Spain \\ 5 Max-Planck-Institut für Astronomie, Königstuhl 17, 69117 Heidelberg, Germany \\ ${ }^{6}$ Landessternwarte, Zentrum für Astronomie der Universität Heidelberg, Königstuhl 12, 69117 Heidelberg, Germany \\ 7 Instituto de Astrofísica de Canarias (IAC), Calle Vía Láctea s/n, 38200, La Laguna Tenerife, Spain \\ ${ }^{8}$ Departamento de Astrofísica, Universidad de La Laguna, 38026, La Laguna Tenerife, Spain \\ 9 Institut für Astrophysik, Georg-August-Universität, Friedrich-Hund-Platz 1, 37077 Göttingen, Germany \\ 10 Departamento de Física de la Tierra y Astrofísica \& IPARCOS-UCM (Instituto de Física de Partículas y del Cosmos de la UCM), \\ Facultad de Ciencias Físicas, Universidad Complutense de Madrid, 28040 Madrid, Spain \\ 11 Institut de Ciències de l'Espai (CSIC-IEEC), Campus UAB, c/ de Can Magrans s/n, 08193 Bellaterra, Barcelona, Spain \\ 12 Institut d'Estudis Espacials de Catalunya (IEEC), 08034 Barcelona, Spain
}

Received 26 January 2021 / Accepted 19 March 2021

\begin{abstract}
Hydrodynamic escape is the most efficient atmospheric mechanism of planetary mass loss and has a large impact on planetary evolution. Three hydrodynamic escape regimes have been identified theoretically: energy-limited, recombination-limited, and photonlimited. However, no evidence of these regimes had been reported until now. Here, we report evidence of these three regimes via an analysis of a helium I triplet at $10830 \AA$ and Ly $\alpha$ absorption involving a 1D hydrodynamic model that allows us to estimate hydrogen recombination and advection rates. In particular, we show that HD 209458 b is in the energy-limited regime, HD $189733 \mathrm{~b}$ is in the recombination-limited regime, and GJ $3470 \mathrm{~b}$ is in the photon-limited regime. These exoplanets can be considered as benchmark cases for their respective regimes.
\end{abstract}

Key words. planets and satellites: atmospheres - planets and satellites: gaseous planets - planets and satellites: individual: GJ $3470 \mathrm{~b}-$ planets and satellites: individual: HD 209458 b - planets and satellites: individual: HD 189733 b

\section{Introduction}

Understanding the diversity, evolution, and formation of planets requires a profound knowledge of their atmospheric escape. This statement especially applies to planets that, at some stage in their history, experience the most effective atmospheric process of mass loss, the hydrodynamic atmospheric escape (also known as blow-off escape). A planetary atmosphere is in hydrodynamic escape when the absorbed stellar high-energy flux is strong enough to produce substantial heating of the atmosphere, which triggers a massive gas outflow, driving the escaping gas to supersonic bulk velocities. This process can lead from a loss of a fraction of the original atmosphere to its complete removal (see e.g. Baraffe et al. 2004, 2005; Muñoz \& Schneider 2019) and hence can shape the current planetary population (e.g. forming the sub-Jovian desert and the evaporation valley; Owen \& Wu 2013; Lopez \& Fortney 2013). Hydrodynamic escape can also significantly change the composition of the primordial atmospheres and thus affects the planetary mass-radius relationship (see e.g. Hu et al. 2015; Malsky \& Rogers 2020).
Several models have been developed for studying planetary atmospheres undergoing hydrodynamic escape (e.g. Watson et al. 1981; Lammer et al. 2003; Yelle 2004; Tian et al. 2005; García-Muñoz 2007; Murray-Clay et al. 2009; Owen \& Alvarez 2016; Salz et al. 2016a; Bourrier \& Lecavelier des Etangs 2013; Shaikhislamov et al. 2020). In particular, Murray-Clay et al. (2009) studied atmospheric mass loss as a function of the incident ultraviolet (UV) flux for a typical hot Jupiter. They found that, under high irradiation, the mass-loss rate was not limited by the radiative energy deposited in the atmosphere, but by the radiation-recombination equilibrium of the escaping gas. When the mass-loss rate is limited by the energy deposition, it scales linearly with the incident UV flux, which is efficiently converted into the work that lifts the gas. When the mass-loss rate is limited by the radiation-recombination equilibrium, it approximately scales to the square root of the incident UV flux since a large fraction of the absorbed energy is lost via radiative cooling. Owen \& Alvarez (2016) found that the mass-loss rate of moderately irradiated low gravity planets can be limited by the number of incoming ionising photons, instead of the absorbed 
energy or the radiation-recombination equilibrium. In this limit, the mass-loss rate scales to the square of the effective absorption radius of the planet. Therefore, it is theoretically possible to identify three distinct hydrodynamic atmospheric escape regimes in hydrogen-dominated atmospheres: energy-limited, recombination-limited, and photon-limited.

This classification is important when using the energylimited approximation for estimating the planetary mass-loss rate (see e.g. Murray-Clay et al. 2009; Bear \& Soker 2011; Owen \& Alvarez 2016; Salz et al. 2016b; Wang \& Dai 2018). This approach, used together with an average heating efficiency of $10-20 \%$ (the most probable range in hydrogen-dominated atmospheres; Shematovich et al. 2014), largely overestimates the mass-loss rate of planets that are in the recombinationlimited regime. It might also lead to significantly inaccurate results if applied to atmospheres in the photon-limited regime as the effective absorption radius could differ considerably from the planetary radius. Hence, the applicability of this approach largely depends on the hydrodynamic escape regime.

Despite the central role of hydrodynamic escape regimes, a lack of suitable observations has prevented their confirmation. While Ly $\alpha$ absorption measurements are promising tracers of this process, they can only be carried out from space and are adversely affected by interstellar absorption and geocoronal emission. This is not the case for the recent observations of the He I $2^{3} \mathrm{~S}-2^{3} \mathrm{P}$ triplet transitions ${ }^{1}$, hereafter $\mathrm{He}\left(2^{3} \mathrm{~S}\right)$ transitions; they provide new opportunities to explore the hydrodynamic atmospheric escape because these lines can be observed with ground-based telescopes and they are not affected by interstellar absorption (e.g. Spake et al. 2018; Nortmann et al. 2018; Allart et al. 2018). Some examples of how these measurements help to better characterise the escaping atmospheres of gas giants were provided by Allart et al. (2018), Mansfield et al. (2018), Lampón et al. (2020, 2021), and Shaikhislamov et al. (2020).

In this work we study the hydrodynamic escape regimes of the giant planets HD 209458 b, HD 189733 b, and GJ $3470 \mathrm{~b}$. The hot Jupiters HD 209458 b and HD 189733 b undergo hydrodynamic escape, as shown by the detection of heavy species escaping in large amounts, such as O I and C II in HD 209458 b as reported by Vidal-Madjar et al. (2004) and O I in HD 189733 b as documented by Ben-Jaffel \& Ballester (2013). GJ 3470 b also undergoes hydrodynamic escape as the outflow bulk radial velocities derived from the $\operatorname{He}\left(2^{3} \mathrm{~S}\right)$ measurements far exceed the sound speed (see Sect. 4.1.3 of Lampón et al. 2021). These planets have different bulk parameters and stellar irradiation levels, which makes studying their escape regimes very interesting.

The Letter is organised as follows. In Sect. 2 we discuss the three regimes that have been theoretically proposed as well as the parameters required to distinguish between them: the ratio of recombination to advection rates and, equivalently, the heating efficiency. In Sect. 3 we summarise the previous modelling and analysis of the three planets on which the calculations of these parameters have been based. In Sect. 4 we present the results and discussion, and in Sect. 5 we provide a summary and our main conclusions.

\section{Criteria to differentiate the hydrodynamic atmospheric escape regimes}

Physically, the three regimes can be distinguished by the production and losses of neutral H (see Murray-Clay et al. 2009;

\footnotetext{
1 At wavelengths $10832.06,10833.22$, and $10833.31 \AA$ in a vacuum; it is often referred to as their air wavelengths of $10830 \AA$.
}

Bear \& Soker 2011; Owen \& Alvarez 2016) as well as by the conversion of the absorbed stellar radiative energy into the work that drives the escape (see Murray-Clay et al. 2009; Salz et al. 2016b).

\subsection{Ratio of recombination to advection rates}

The steady-state continuity equation of neutral $\mathrm{H}$ species, $\mathrm{H}^{0}$, can be expressed as (see Appendix A)

$-v_{\mathrm{r}} \frac{\partial f_{\mathrm{H}^{0}}}{\partial r}+f_{\mathrm{H}^{+}} n_{\mathrm{H}^{+}} \alpha_{\mathrm{H}}-f_{\mathrm{H}^{0}} J_{\mathrm{H}}=0$,

where $r$ is the distance from the centre of the planet; $v_{\mathrm{r}}$ is the bulk radial velocity of the gas; $f_{\mathrm{H}^{0}}$ and $f_{\mathrm{H}^{+}}$are the mole fractions of $\mathrm{H}^{0}$ and the ionised $\mathrm{H}, \mathrm{H}^{+}$, respectively; $n_{\mathrm{H}^{+}}$is the $\mathrm{H}^{+}$number density; and $\alpha_{\mathrm{H}}$ and $J_{\mathrm{H}}$ are the $\mathrm{H}$ recombination and photoionisation rates. We define the recombination production rate as $P_{\mathrm{rec}}=f_{\mathrm{H}^{+}} n_{\mathrm{H}^{+}} \alpha_{\mathrm{H}}$ and the advection rate as $P_{\mathrm{adv}}=-v_{\mathrm{r}} \partial f_{\mathrm{H}^{0}} / \partial r$.

Similarly, and like Owen \& Alvarez (2016), we consider the ionisation front (IF) as the region where the atmosphere is partially ionised, that is, from the altitude where $f_{\mathrm{H}^{0}}$ is close to one $-f_{\mathrm{H}^{0}} \geq 0.95$, where the optical depth of the X-ray and extreme $\mathrm{UV}$ (XUV) radiation, $\tau_{\mathrm{XUV}}$, is $\geq 1-$ up to the altitude where the atmosphere is almost fully ionised $-f_{\mathrm{H}^{0}} \leq 0.05$ and $\tau_{\mathrm{XUV}} \ll 1$. In this region, the photo-ionisation rate is greater than the recombination rate and advection is non-negligible.

Following Murray-Clay et al. (2009) and Owen \& Alvarez (2016), we characterise the different hydrodynamic atmospheric escape regimes as follows: (1) In the recombination-limited regime, the IF region is very narrow compared with the scale of the flow. The gas is almost in radiation-recombination equilibrium (i.e. advection is negligible, $P_{\mathrm{rec}} / P_{\mathrm{adv}} \gg 1$ ) in practically all of the upper atmosphere. (2) In the photon-limited regime, the photo-ionisation rate is much faster than the recombination rate such that the recombination process is negligible in the whole upper atmosphere and $P_{\mathrm{rec}} / P_{\mathrm{adv}} \ll 1$. Consequently, the IF region occupies the whole upper atmosphere of the planet. (3) The energy-limited regime is an intermediate case in between the recombination- and the photon-limited regimes. The IF is wide, although it does not encompass the entire flow as in the photonlimited regime. The advection and the recombination rates are non-negligible in the whole upper atmosphere.

\subsection{Heating efficiency criterion}

Although the ratio of recombination to advection rates provides us with a direct way of determining the hydrodynamic escape regime, it is also useful to look at these ratios from the point of view of heating efficiency, as previously done by several authors. This has the advantage of providing us with better insight into the physical processes behind the escape. The heating efficiency, $\eta$, is usually defined as the fraction of the absorbed stellar radiative energy, $W_{\mathrm{hv}}$, which is converted into the kinetic energy of the gas, namely,

$\eta=\frac{W_{\mathrm{hv}}-W_{\mathrm{e}}-W_{\text {cool }}}{W_{\mathrm{hv}}}$,

where $W_{\mathrm{e}}$ is the rate of energy lost by photo-ionisation and photo-electron impact processes (as the excitation and ionisation of atoms by collisions with photoelectrons; see e.g. Shematovich et al. 2014) and $W_{\text {cool }}$ is the radiative cooling rate, mostly produced by Ly $\alpha$ and free-free emission ( $\mathrm{Salz}$ et al. $2016 \mathrm{~b})$. Thus, $W_{\mathrm{hv}}-W_{\mathrm{e}}-W_{\text {cool }}$ represents the energy from 
the absorbed stellar radiation that is converted into the kinetic energy of the gas species (atoms, ions, and electrons), the socalled thermal heating.

In the energy- and photon-limited regimes, radiative cooling is moderate or negligible, while in the recombination-limited regime it is a considerable fraction of the absorbed energy. If radiative cooling is neglected, the heating efficiency of stellar XUV radiation is about $0.1-0.2$ in hydrogen-dominated planetary upper atmospheres (see e.g. Shematovich et al. 2014; Salz et al. 2016b). Consequently, the heating efficiency of the energy-limited and the photon-limited regimes is expected to be in the range of $0.1-0.2$. In contrast, the heating efficiency of the recombination-limited regime is expected to be much less than 0.1 (see e.g. Salz et al. 2016b).

\section{Recombination and advection rates and heating efficiencies}

The recombination and advection rates of $\mathrm{H}^{0}$ and the heating efficiencies analysed here are based on the modelling of the observations of the $\mathrm{He}\left(2^{3} \mathrm{~S}\right)$ absorption of HD $209458 \mathrm{~b}, \mathrm{HD} 189733 \mathrm{~b}$, and GJ $3470 \mathrm{~b}$ that were performed by Lampón et al. (2020, 2021). They used a 1D hydrodynamic model with a non-local thermodynamic equilibrium treatment for the $\operatorname{He}\left(2^{3} \mathrm{~S}\right)$ state, and, by comparing the simulated and observed $\mathrm{He}\left(2^{3} \mathrm{~S}\right)$ absorption, they derived well-constrained relationships and narrow intervals between the mass-loss rate, $\dot{M}$, and the temperature, $T$, in the upper atmosphere of these exoplanets. Additionally, by comparing the $\mathrm{H}^{0}$ density distribution with those derived from Ly $\alpha$ measurements, they constrained the $\mathrm{H} / \mathrm{He}$ mole-fraction ratio.

The $\mathrm{He}\left(2^{3} \mathrm{~S}\right)$ production is controlled by electron concentration via recombination with $\mathrm{He}^{+}$as well as by electron collision excitation of $\mathrm{He}\left(1^{1} \mathrm{~S}\right)$ (see Oklopčić \& Hirata 2018; Lampón et al. 2020). As electrons are predominantly produced by the photo-ionisation of $\mathrm{H}$ and lost by advection and $\mathrm{H}^{+}$ recombination, the $\mathrm{He}\left(2^{3} \mathrm{~S}\right)$ concentration profile is thus mainly controlled by the $\mathrm{H}$ production and loss rates. Therefore, by constraining the $\mathrm{He}\left(2^{3} \mathrm{~S}\right)$ concentration profile, as was done by Lampón et al. (2020, 2021), we simultaneously constrain the production and loss rates of $\mathrm{H}$. Hence, in this work we analysed the production and loss rates of $\mathrm{H}$ corresponding to the $T-\dot{M}$ ranges and $\mathrm{H} / \mathrm{He}$ ratios of $99 / 1$ for $\mathrm{HD} 189733 \mathrm{~b}$ and $98 / 2$ for HD 209458 b and GJ 3470 b, which were previously obtained by Lampón et al. $(2020,2021)$ and are summarised in Fig. 1.

\section{Results and discussion}

In this section we discuss the hydrodynamic escape regimes on the basis of the $\mathrm{H}^{0}$ and $\mathrm{H}^{+}$distributions as well as on the basis of the heating efficiencies for the three planets.

\subsection{Structure, production, and losses of the neutral $H$}

The $\mathrm{H}^{0}$ mole fractions that result from the derived $T-\dot{M}$ ranges (see the symbols in Fig. 1) are shown in Fig. 2. The $f_{\mathrm{H}^{0}}$ profiles for HD $189733 \mathrm{~b}$ show a very narrow IF; it is confined at the lower boundary (set at $1.02 R_{\mathrm{P}}$ for all three planets), well below its Roche lobe, which is located at $3.0 R_{\mathrm{P}}$. Accordingly, this planet shows a wide, fully ionised region. In contrast, GJ $3470 \mathrm{~b}$ shows a very extended IF, from about $1.2 R_{\mathrm{P}}$ to far beyond its Roche lobe at $3.6 R_{\mathrm{P}}$. Therefore, no atmospheric region of GJ $3470 \mathrm{~b}$ is fully ionised. The $\mathrm{H}^{0}$ structure of HD $209458 \mathrm{~b}$ shows a relatively wide IF, extending from the lower boundary

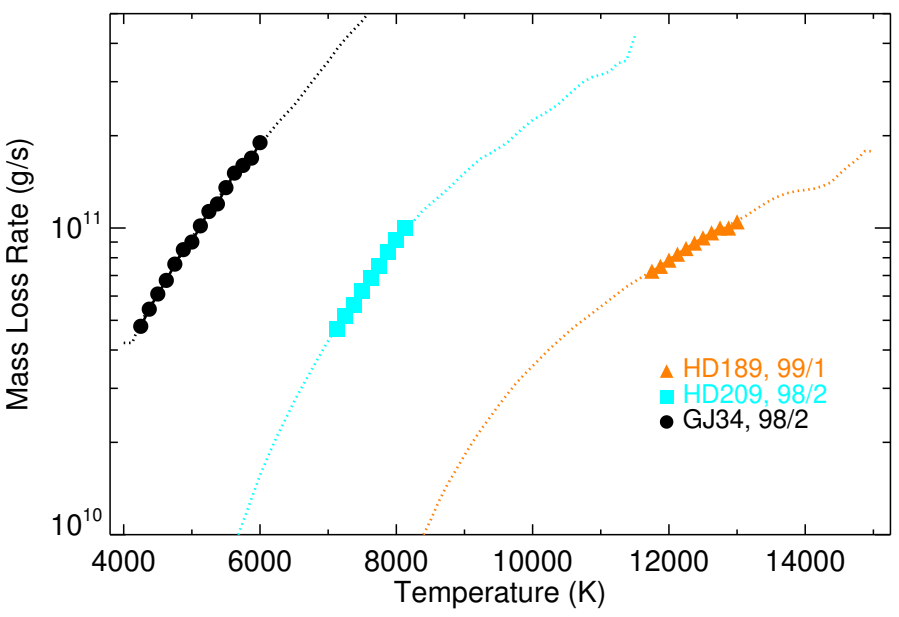

Fig. 1. Ranges of temperature and mass-loss rates for HD 209458 b, HD 189733 b, and GJ 3470 b for $\mathrm{H} / \mathrm{He}$ ratios of $98 / 2$, 99/1, and $98 / 2$, respectively, derived by Lampón et al. $(2020,2021)$. Symbols show the derived ranges and dotted lines the extended ranges (see Sect. 4.1).

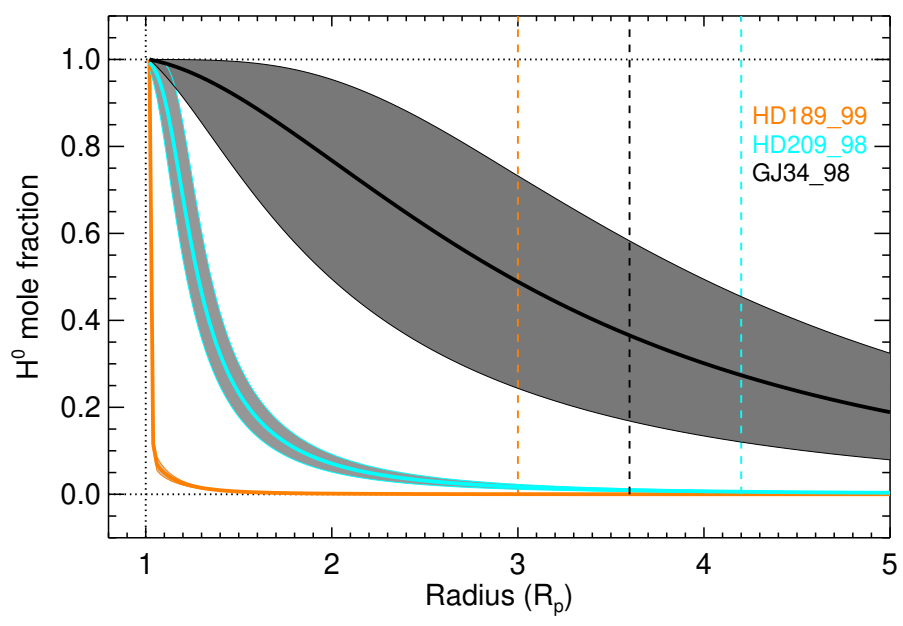

Fig. 2. Neutral $\mathrm{H}$ mole fraction profiles resulting from the measured $\mathrm{He}\left(2^{3} \mathrm{~S}\right)$ and Ly $\alpha$ absorption spectra for HD $189733 \mathrm{~b}$, HD $209458 \mathrm{~b}$, and GJ $3470 \mathrm{~b}$ with the representative $\mathrm{H} / \mathrm{He}$ ratios of $99 / 1,98 / 2$, and $98 / 2$, respectively. The grey shaded areas correspond to the temperature and mass-loss rate ranges (symbols) in Fig. 1. The thicker solid lines are the mean profiles. The vertical dashed lines indicate the mean Roche lobes.

to about $2.3 R_{\mathrm{P}}$, which is below its Roche lobe (at $4.2 R_{\mathrm{P}}$ ); this IF is thus wider than that of HD $189733 \mathrm{~b}$ and narrower than that of GJ 3470 b. Therefore, as in HD 189733 b, there is a region that is almost fully ionised below the Roche lobe, although it is narrower than the corresponding region in HD $189733 \mathrm{~b}$.

Figure 3 shows the ratio of recombination to advection terms, $P_{\text {rec }} / P_{\text {adv }}$, for the derived $T-\dot{M}$ range of the measured $\mathrm{He}\left(2^{3} \mathrm{~S}\right)$ and Ly $\alpha$ absorption spectra for HD 209458 b, HD 189733 b, and GJ 3470 b (see Fig. 1). For the case of HD 189733 b, recombination dominates in practically the whole upper atmosphere. In contrast, the $\mathrm{H}^{0}$ density is dominated by advection in the whole upper atmosphere of GJ 3470 b. In HD 209458 b, the $\mathrm{H}^{0}$ density is dominated by advection $\left(P_{\mathrm{rec}} / P_{\mathrm{adv}}<1\right)$ in the lower part of the IF, but by recombination $\left(P_{\mathrm{rec}} / P_{\mathrm{adv}}>1\right)$ in the upper part of the IF and above. That is, neither advection nor recombination rates are negligible in the upper atmosphere of HD 209458 b. Hence, according to the criteria discussed in Sect.2.1, these results show that HD $189733 \mathrm{~b}$ is in the recombination-limited regime, 

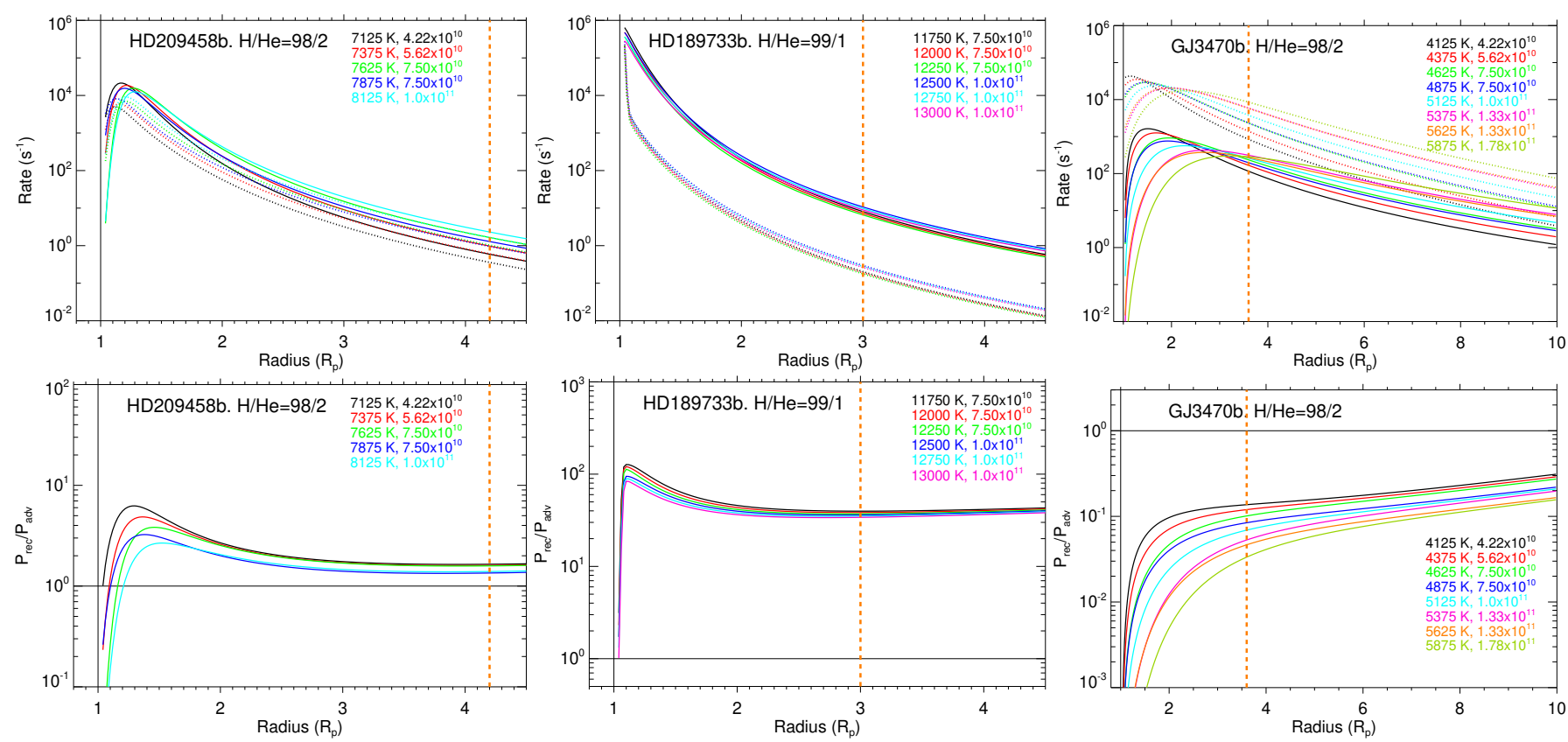

Fig. 3. Recombination and advection for HD 209458 b, HD 189733 b, and GJ 3470 b. Upper row: production rates of H by recombination (solid lines) and advection (dashed lines) derived from the fits of the measured $\mathrm{He}\left(2^{3} \mathrm{~S}\right)$ absorption spectra (see symbols in Fig. 1) in the atmospheres of HD 209458 b (left), HD 189733 b (middle), and GJ 3470 b (right) and the representative H/He ratios of 98/2, 99/1, and 98/2, respectively. Lower row: ratios of the recombination to the advection. The vertical dashed orange lines indicate the mean Roche lobes. The different scales of the $\mathrm{x}$ and $y$ axes for the different planets should be noted.

GJ $3470 \mathrm{~b}$ is in the photon-limited regime, and HD $209458 \mathrm{~b}$ is in the energy-limited regime.

We also analysed other plausible $T-\dot{M}$ values beyond the derived interval, hereafter the 'extended' $T-\dot{M}$ range (see the dotted lines in Fig. 1). These $T-\dot{M}$ values are still compatible with the measured $\operatorname{He}\left(2^{3} \mathrm{~S}\right)$ and $\operatorname{Ly} \alpha$ absorption; however, they are less likely as they yield a higher $\chi^{2}$. With this, we aim to determine if they would affect the classification with respect to the escape regime. Figures B. 1 and B. 3 show the results for the $P_{\text {rec }} / P_{\text {adv }}$ ratios and the corresponding $\mathrm{H}^{0}$ mole fractions, respectively. We found that the extended $T-\dot{M}$ ranges for HD $189733 \mathrm{~b}$ and GJ $3470 \mathrm{~b}$ exhibit the same behaviour as that of the corresponding derived $T-\dot{M}$ ranges (see Fig. 3). In contrast, production terms for HD $209458 \mathrm{~b}$ at temperatures below $\sim 7000 \mathrm{~K}$ (see Fig. B.1) are at the limit between the energy-limited and recombination-limited regimes, with advection becoming less important relative to recombination. However, when comparing the $\mathrm{H}$ density profiles for these conditions with those derived from the Ly $\alpha$ measurements by García-Muñoz (2007), Koskinen et al. (2013), and Salz et al. (2016a), we found that the $\mathrm{H}^{0}$ density profiles are significantly smaller. We thus conclude that the hydrodynamic regimes discussed above for the three planets are not altered when considering the extended $T-\dot{M}$ ranges. We find that the classification of the escape regimes also remains unchanged in these exoplanets if the commonly used $\mathrm{H} / \mathrm{He}$ fraction of $90 / 10$ is assumed instead of the ratios derived from $\mathrm{He}\left(2^{3} \mathrm{~S}\right)$ and Ly $\alpha$ transmission spectroscopy (see Figs. B.2 and B.4).

For HD 189733 b, we used an XUV flux that accounts for several small flares to provide an average model of active and non-active periods (see Sect. 3.2 in Lampón et al. 2021). In order to explore the hydrodynamic regime of this planet in quiescent stages, we conducted a test using the XUV flux from the X-exoplanets model in Sanz-Forcada et al. (2011), which is about a factor of three smaller than the one used here. We found that the hydrodynamic regime remains recombination-limited despite such a considerable XUV flux decrease.

\subsection{Heating efficiencies}

To calculate the heating efficiencies of the upper atmospheres of the planets, we followed the method from Salz et al. (2016b). Essentially, we used the relationship between the heating efficiency, $\eta$, and the mass-loss rate for the energy-limited approximation, $\dot{M}_{\mathrm{EL}}$ (Watson et al. 1981; Erkaev et al. 2007), together with the relationship $\dot{M} / \dot{M}_{\mathrm{EL}}=4 / 5$ derived by Salz et al. (2016b) to obtain

$\dot{M}=\frac{4}{5} \frac{4 \pi R_{\mathrm{p}} R_{\mathrm{XUV}}^{2} F_{\mathrm{XUV}}}{G K(\xi) M_{\mathrm{P}}} \eta$,

where $M_{\mathrm{p}}$ and $R_{\mathrm{P}}$ are the planetary mass and radius, respectively; $F_{\mathrm{XUV}}$ is the XUV stellar flux at the planetary orbital separation; $R_{\mathrm{XUV}}$ is the effective absorption radius, the altitude where the XUV optical depth is unity; and $G$ is the gravitational constant. Here, $K(\xi)=1-1.5 \xi+0.5 \xi^{3}$ is the potential energy reduction factor, with $\xi=\left(M_{\mathrm{P}} / M_{\star}\right)^{1 / 3}\left(a / R_{\mathrm{P}}\right)$, where $a$ is the planetary orbital separation and $M_{\star}$ the stellar mass. Therefore, taking the $\dot{M}$ and $R_{\text {XUV }}$ obtained from the $\mathrm{He}\left(2^{3} \mathrm{~S}\right)$ measurements and the required system parameters from Table B.1, we calculated their corresponding $\eta$ values (see Fig. 4). The $\dot{M}$ values are taken from Fig. 1 (ranges limited by the symbols), and the $R_{\mathrm{XUV}}$ values are $\approx 1.02 R_{\mathrm{P}}$ for HD $189733 \mathrm{~b}$ (i.e. the lower boundary), about $1.16-$ $1.30 R_{\mathrm{P}}$ for HD $209458 \mathrm{~b}$, and about $1.38-2.46 R_{\mathrm{P}}$ for GJ $3470 \mathrm{~b}$.

As shown by the thick lines in Fig. 4, $\eta$ reaches high values in HD 209458 b and GJ 3470 b but remains very low $(\eta<0.04)$ for HD $189733 \mathrm{~b}$. This indicates that HD $189733 \mathrm{~b}$ is in the recombination-limited regime, as predicted (e.g. by Salz et al. 2016b).

We note that the derived $T-\dot{M}$ range for HD $209458 \mathrm{~b}$ was obtained by limiting the heating efficiency to the range of 
M. Lampón et al.: Evidence of energy-, recombination-, and photon-limited escape regimes

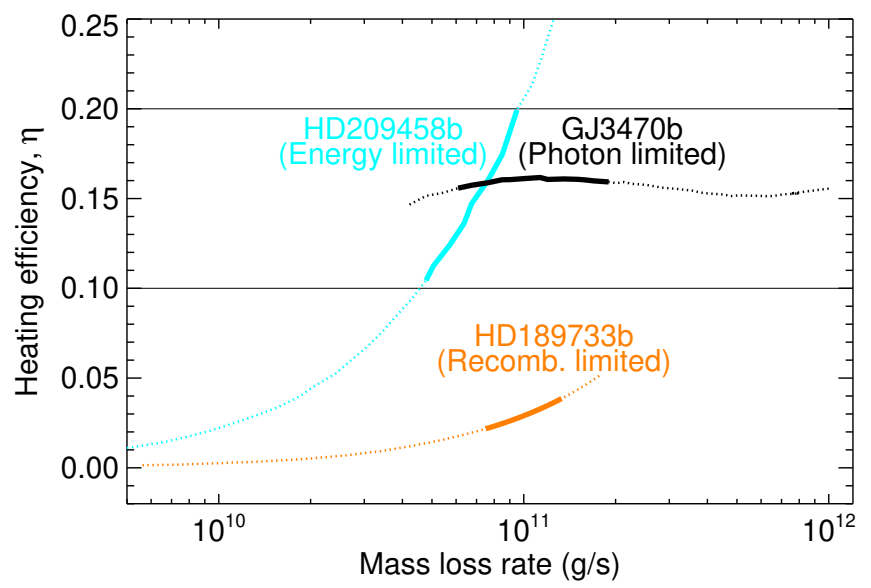

Fig. 4. Heating efficiency, $\eta$, versus mass-loss rates for HD 189733 b (orange), HD 209458 b (cyan), and GJ $3470 \mathrm{~b}$ (black). H/He ratios of 99/1 for HD 189733 b and of 98/2 for HD 209458 b and GJ $3470 b$ were used. Dotted lines show the extended $T-\dot{M}$ ranges and thick lines the derived $T-\dot{M}$ ranges (see the dotted lines and symbols, respectively, in Fig. 1).

0.1-0.2 (Lampón et al. 2020), as shown in Fig. 4. Values of $\eta \leq 0.1$ would indicate a recombination-limited regime (see Sect. 2.2). However, they correspond to temperatures below $T \approx$ $7000 \mathrm{~K}$, which, as discussed in Sect. 4.1, were discarded on the basis of Ly $\alpha$ observations. This as well as the heating efficiency argument led us to conclude that HD 209458b is not in the recombination-limited regime.

Heating efficiency remains almost constant with $\dot{M}$ in GJ 3470 b, in contrast to HD 209458 b. As the upper atmosphere of GJ 3470 b is highly extended, an increase in $\dot{M}$ implies higher $R_{\text {XUV }}$ (i.e. a higher planetary XUV cross-section), which prevents $\eta$ from increasing. The fact that $\eta$ is nearly constant is equivalent to stating that $\dot{M} \propto R_{\text {XUV }}^{2}$ (see Eq. (3)), which, according to Owen \& Alvarez (2016), indicates that GJ 3470 b is in the photon-limited regime.

\section{Summary}

Several theoretical studies have identified three distinct hydrodynamic atmospheric escape regimes in hydrogen-dominated planetary atmospheres: energy-limited, recombination-limited, and photon-limited. In addition to the importance from a theoretical point of view, the classification of the hydrodynamic escape regimes has important consequences for the estimation of the mass-loss rate by models based on the energy-limited approach. However, no evidence of these regimes had been reported to date.

In this work, we studied the recombination to advection rates of the upper atmospheric $\mathrm{H}$ obtained in a previous analysis of the measured absorption spectrum of the He I triplet lines at $10830 \AA$ and Ly $\alpha$ of HD 189733 b, HD 209458 b, and GJ 3470 b, which was performed by Lampón et al. (2020, 2021). Furthermore, based on these results, we also calculated the heating efficiencies, following Salz et al. (2016b).

We found that in HD $189733 \mathrm{~b}$ the neutral $\mathrm{H}$ production is governed by recombination in the whole upper atmosphere, giving rise to a very narrow IF. In contrast, advection dominates the neutral $\mathrm{H}$ density in the entire upper atmosphere of GJ $3470 \mathrm{~b}$, leading to a very extended IF. HD $209458 \mathrm{~b}$ is an intermediate case between HD $189733 \mathrm{~b}$ and GJ $3470 \mathrm{~b}$ : the advection and the recombination processes are non-negligible, which forms a relatively extended IF.

The derived heating efficiencies for HD 209458b and GJ $3470 \mathrm{~b}$ are similar to those expected from heating via photoelectrons, while those of HD $189733 \mathrm{~b}$ are much lower. Therefore, HD 209458 b and GJ 3470 b show little loss by radiative cooling, in contrast to HD 189733 b. Additionally, we found that $\dot{M} \propto R_{\text {XUV }}^{2}$ in GJ 3470 b, which leads to a nearly constant heating efficiency in the range of $0.15-0.17$. Following two criteria, one based on the productions and losses of the neutral $\mathrm{H}$ and the other based on energy balance considerations, we have shown that HD $189733 \mathrm{~b}$ is in the recombination-limited regime, GJ $3470 \mathrm{~b}$ is in the photon-limited regime, and HD 209458 b is in the energy-limited regime.

Our analysis involves a series of important model assumptions, such as the use of 1D models without an explicit solution of the energy budget equation and assumed lower boundary conditions. We have shown that these assumptions do not significantly affect the derived bulk properties, such as thermospheric temperatures, mass-loss rates, radial velocities, $\mathrm{H} / \mathrm{He}$ ratios, and, in particular, the recombination and advection rates. Consequently, we are confident that our analysis of measured $\mathrm{He}\left(2^{3} \mathrm{~S}\right)$ and $\mathrm{Ly} \alpha$ absorption provides robust evidence for the existence of the three theoretically predicted hydrodynamic escape regimes in hydrogen-dominated atmospheres. Accordingly, HD 209458 b, HD 189733 b, and GJ 3470 b can be considered as benchmark cases for their respective regimes.

Acknowledgements. We thank the referee for very useful comments. CARMENES is an instrument for the Centro Astronómico Hispano-Alemán (CAHA) at Calar Alto (Almería, Spain), operated jointly by the Junta de Andalucía and the Instituto de Astrofísica de Andalucía (CSIC). CARMENES was funded by the Max-Planck-Gesellschaft (MPG), the Consejo Superior de Investigaciones Científicas (CSIC), the Ministerio de Economía y Competitividad (MINECO) and the European Regional Development Fund (ERDF) through projects FICTS-2011-02, ICTS-2017-07-CAHA-4, and CAHA16CE-3978, and the members of the CARMENES Consortium (Max-PlanckInstitut für Astronomie, Instituto de Astrofísica de Andalucía, Landessternwarte Königstuhl, Institut de Ciències de 1'Espai, Institut für Astrophysik Göttingen, Universidad Complutense de Madrid, Thüringer Landessternwarte Tautenburg, Instituto de Astrofísica de Canarias, Hamburger Sternwarte, Centro de Astrobiología and Centro Astronómico Hispano-Alemán), with additional contributions by the MINECO, the Deutsche Forschungsgemeinschaft through the Major Research Instrumentation Programme and Research Unit FOR2544 "Blue Planets around Red Stars", the Klaus Tschira Stiftung, the states of Baden-Württemberg and Niedersachsen, and by the Junta de Andalucía. We acknowledge financial support from the Agencia Estatal de Investigación of the Ministerio de Ciencia, Innovación y Universidades and the ERDF through projects ESP2016-76076-R, ESP2017-87143R, BES-2015-074542, PID2019-110689RB-I00/AEI/10.13039/501100011033, PGC2018-099425-B-I00, PID2019-109522GB-C51/2/3/4, PGC2018-098153B-C33, AYA2016-79425-C3-1/2/3-P, ESP2016-80435-C2-1-R, and the Centre of Excellence "Severo Ochoa" and "María de Maeztu" awards to the Instituto de Astrofísica de Canarias (SEV-2015-0548), Instituto de Astrofísica de Andalucía (SEV-2017-0709), and Centro de Astrobiología (MDM-2017-0737), and the Generalitat de Catalunya/CERCA programme. T.H. acknowledges support from the European Research Council under the Horizon 2020 Framework Program via the ERC Advanced Grant Origins 832428. A.S.L. acknowledges funding from the European Research Council under the European Union's Horizon 2020 research and innovation program under grant agreement No 694513.

\section{References}

Agol, E., Cowan, N. B., Knutson, H. A., et al. 2010, ApJ, 721, 1861 Allart, R., Bourrier, V., Lovis, C., et al. 2018, Science, 362, 1384 Baluev, R. V., Sokov, E. N., Shaidulin, V. S., et al. 2015, MNRAS, 450, 3101 Baraffe, I., Selsis, F., Chabrier, G., et al. 2004, A\&A, 419, L13

Baraffe, I., Chabrier, G., Barman, T. S., et al. 2005, A\&A, 436, L47 Bear, E., \& Soker, N. 2011, MNRAS, 414, 1788

Ben-Jaffel, L., \& Ballester, G. E. 2013, A\&A, 553, A52 
Bonfils, X., Gillon, M., Udry, S., et al. 2012, A\&A, 546, A27

Bourrier, V., \& Lecavelier des Etangs, A. 2013, A\&A, 557, A124

Bourrier, V., Lecavelier des Etangs, A., Ehrenreich, D., et al. 2018, A\&A, 620, A 147

de Kok, R. J., Brogi, M., Snellen, I. A. G., et al. 2013, A\&A, 554, A82

Eggleton, P. P. 1983, ApJ, 268, 368

Erkaev, N. V., Kulikov, Y. N., Lammer, H., et al. 2007, A\&A, 472, 329

García-Muñoz, A. 2007, Planet. Space Sci., 55, 1426

Hu, R., Seager, S., \& Yung, Y. L. 2015, ApJ, 807, 8

Koskinen, T., Harris, M., Yelle, R., \& Lavvas, P. 2013, Icarus, 226, 1678

Lammer, H., Selsis, F., Ribas, I., et al. 2003, ApJ, 598, L121

Lampón, M., López-Puertas, M., Lara, L. M., et al. 2020, A\&A, 636, A13

Lampón, M., López-Puertas, M., Sanz-Forcada, J., et al. 2021, A\&A, 647, A129

Lopez, E. D., \& Fortney, J. J. 2013, ApJ, 776, 2

Malsky, I., \& Rogers, L. A. 2020, ApJ, 896, 48

Mansfield, M., Bean, J. L., Oklopčić, A., et al. 2018, ApJ, 868, L34

Muñoz, A. G., \& Schneider, P. C. 2019, ApJ, 884, L43

Murray-Clay, R. A., Chiang, E. I., \& Murray, N. 2009, ApJ, 693, 23

Nortmann, L., Palle, E., Salz, M., et al. 2018, Science, 362, 1388
Oklopčić, A., \& Hirata, C. M. 2018, ApJ, 855, L11

Owen, J. E., \& Alvarez, M. A. 2016, ApJ, 816, 34

Owen, J. E., \& Wu, Y. 2013, ApJ, 775, 105

Pallé, E., Nortmann, L., Casasayas-Barris, N., et al. 2020, A\&A, 638, A61

Salz, M., Czesla, S., Schneider, P. C., \& Schmitt, J. H. M. M. 2016a, A\&A, 586, A75

Salz, M., Schneider, P. C., Czesla, S., \& Schmitt, J. H. M. M. 2016b, A\&A, 585, L2

Sanz-Forcada, J., Micela, G., Ribas, I., et al. 2011, A\&A, 532, A6

Shaikhislamov, I. F., Khodachenko, M. L., Lammer, H., et al. 2020, MNRAS, 500, 1404

Shematovich, V. I., Ionov, D. E., \& Lammer, H. 2014, A\&A, 571, A94

Spake, J. J., Sing, D. K., Evans, T. M., et al. 2018, Nature, 557, 68

Tian, F., Toon, O. B., Pavlov, A. A., \& De Sterck, H. 2005, ApJ, 621, 1049

Torres, G., Winn, J. N., \& Holman, M. J. 2008, ApJ, 677, 1324

Vidal-Madjar, A., Désert, J. M., des Etangs, A. L., et al. 2004, ApJ, 604, 69

Wang, L., \& Dai, F. 2018, ApJ, 860, 175

Watson, A. J., Donahue, T. M., \& Walker, J. C. 1981, Icarus, 48, 150

Yelle, R. V. 2004, Icarus, 170, 167 
M. Lampón et al.: Evidence of energy-, recombination-, and photon-limited escape regimes

\section{Appendix A: The steady-state continuity equation of neutral $H$}

The hydrodynamic continuity equation of mass can be expressed as

$\dot{M}=4 \pi r^{2} \rho_{\mathrm{r}} v_{\mathrm{r}}$

where $\rho_{\mathrm{r}}=n_{\mathrm{H}} m_{\mathrm{H}}+n_{\mathrm{He}} m_{\mathrm{He}} ; n_{\mathrm{H}}$ and $n_{\mathrm{He}}$ are the number densities of $\mathrm{H}$ and $\mathrm{He}$, respectively; and $m_{\mathrm{H}}$ and $m_{\mathrm{He}}$ are the atomic masses of $\mathrm{H}$ and $\mathrm{He}$, respectively. Assuming that $n_{\mathrm{H}} / n_{\mathrm{He}}$ (hereafter $\mathrm{H} / \mathrm{He}$ ) is constant with respect to $r$, then $n_{\mathrm{He}}=(\mathrm{H} / \mathrm{He})^{-1} n_{\mathrm{H}}$. Therefore, $\rho_{\mathrm{r}}=n_{\mathrm{H}} x$, where $x=m_{\mathrm{H}}+$ $(\mathrm{H} / \mathrm{He})^{-1} m_{\mathrm{He}}$, is constant. As such, the mass-loss rate can be expressed as

$\dot{M}=4 \pi r^{2} n_{\mathrm{H}} x v_{\mathrm{r}}$.

The continuity equation of $\mathrm{H}^{0}$ can be written as

$\frac{1}{r^{2}} \frac{\partial\left(r^{2} n_{\mathrm{H}^{0}} v_{\mathrm{r}}\right)}{\partial r}=n_{\mathrm{H}^{+}} n_{\mathrm{e}} \alpha_{\mathrm{H}}-n_{\mathrm{H}^{0}} J_{\mathrm{H}}$ and, deriving, as

$\frac{2 n_{\mathrm{H}^{0}} v_{\mathrm{r}}}{r}+v_{\mathrm{r}} \frac{\partial n_{\mathrm{H}^{0}}}{\partial r}+n_{\mathrm{H}^{0}} \frac{\partial v_{\mathrm{r}}}{\partial r}=n_{\mathrm{H}^{+}} n_{\mathrm{e}} \alpha_{\mathrm{H}}-n_{\mathrm{H}^{0}} J_{\mathrm{H}}$.

Taking $v_{\mathrm{r}}=\dot{M} /\left(4 \pi r^{2} n_{\mathrm{H}} x\right)$ from Eq. (A.2) and re-arranging Eq. (A.4), we have

$v_{\mathrm{r}} \frac{1}{n_{\mathrm{H}}}\left[n_{\mathrm{H}} \frac{\partial n_{\mathrm{H}^{0}}}{\partial r}-n_{\mathrm{H}^{0}} \frac{\partial n_{\mathrm{H}}}{\partial r}\right]=n_{\mathrm{H}^{+}} n_{\mathrm{e}} \alpha_{\mathrm{H}}-n_{\mathrm{H}^{0}} J_{\mathrm{H}}$.

Taking into account that

$\frac{\partial f_{\mathrm{H}^{0}}}{\partial r}=\frac{\partial\left(n_{\mathrm{H}^{0}} / n_{\mathrm{H}}\right)}{\partial r}=\frac{1}{n_{\mathrm{H}}^{2}}\left(n_{\mathrm{H}} \frac{\partial n_{\mathrm{H}^{0}}}{\partial r}-n_{\mathrm{H}^{0}} \frac{\partial n_{\mathrm{H}}}{\partial r}\right)$

and including it in Eq. (A.5), we obtain

$v_{\mathrm{r}} n_{\mathrm{H}} \frac{\partial f_{\mathrm{H}^{0}}}{\partial r}=n_{\mathrm{H}^{+}} n_{\mathrm{e}} \alpha_{\mathrm{H}}-n_{\mathrm{H}^{0}} J_{\mathrm{H}}$.

Assuming $n_{\mathrm{e}}=n_{\mathrm{H}^{+}}$, and re-arranging Eq. (A.7), we finally obtain Eq. (1). 


\section{Appendix B: Other tables and figures}

In Table B.1 we provide the most relevant system parameters used in our calculations. In Figs. B.1-B.4 we show ratios of recombination to advection and $\mathrm{H}^{0}$ mole fraction profiles for the extended $T-\dot{M}$ ranges (dotted lines in Fig. 1) as well as for the $\mathrm{H} / \mathrm{He}$ composition of $90 / 10$ (see the symbols in Fig. 5 of Lampón et al. 2021).
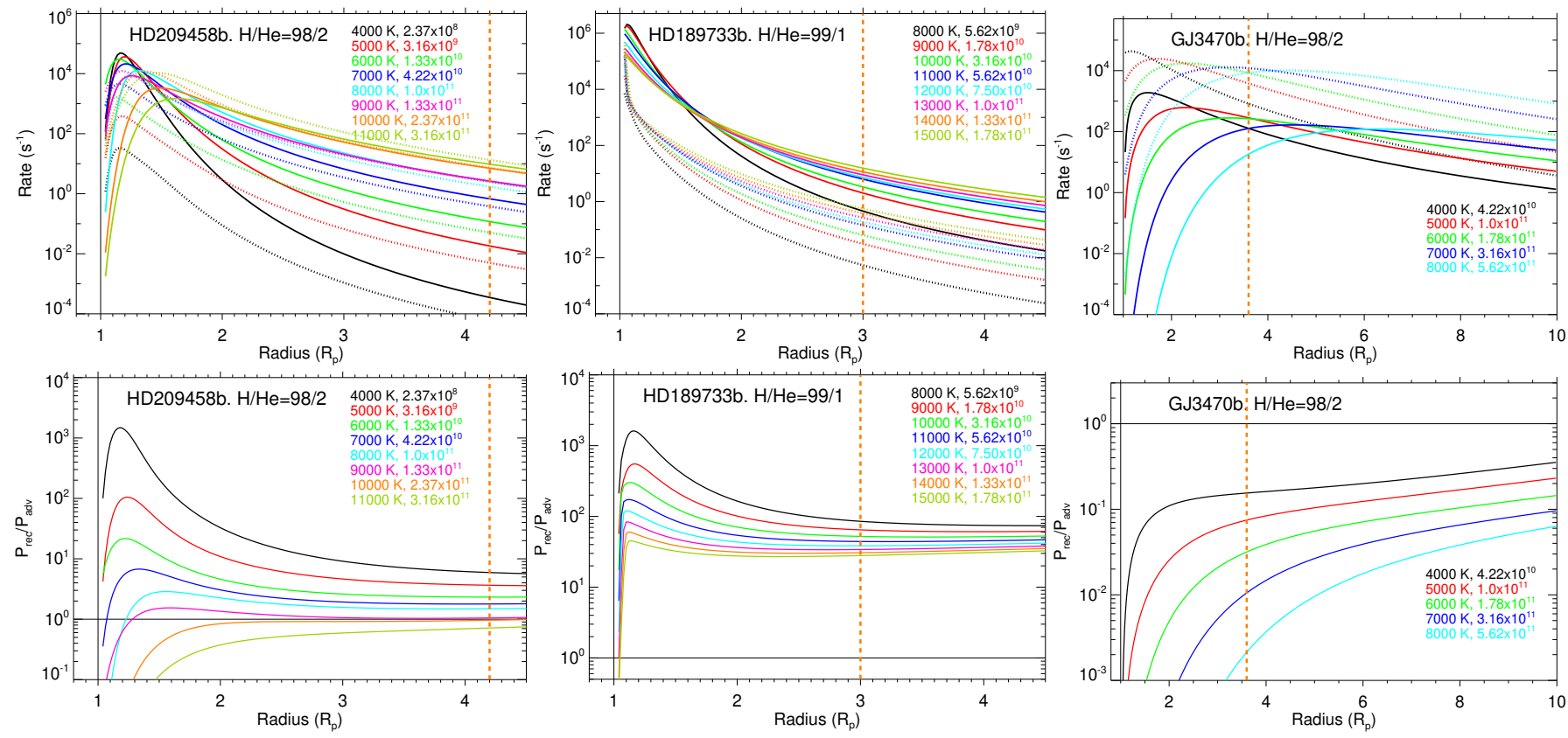

Fig. B.1. Same as Fig. 3 but for the extended $T-\dot{M}$ ranges (see the dotted lines in Fig. 1). Upper row: rates of $\mathrm{H}$ by recombination (solid lines) and advection (dotted lines). Lower row: ratios of recombination to advection. The vertical dashed orange lines indicate the mean Roche lobes. We note the different scales of the $x$ and $y$ axes.
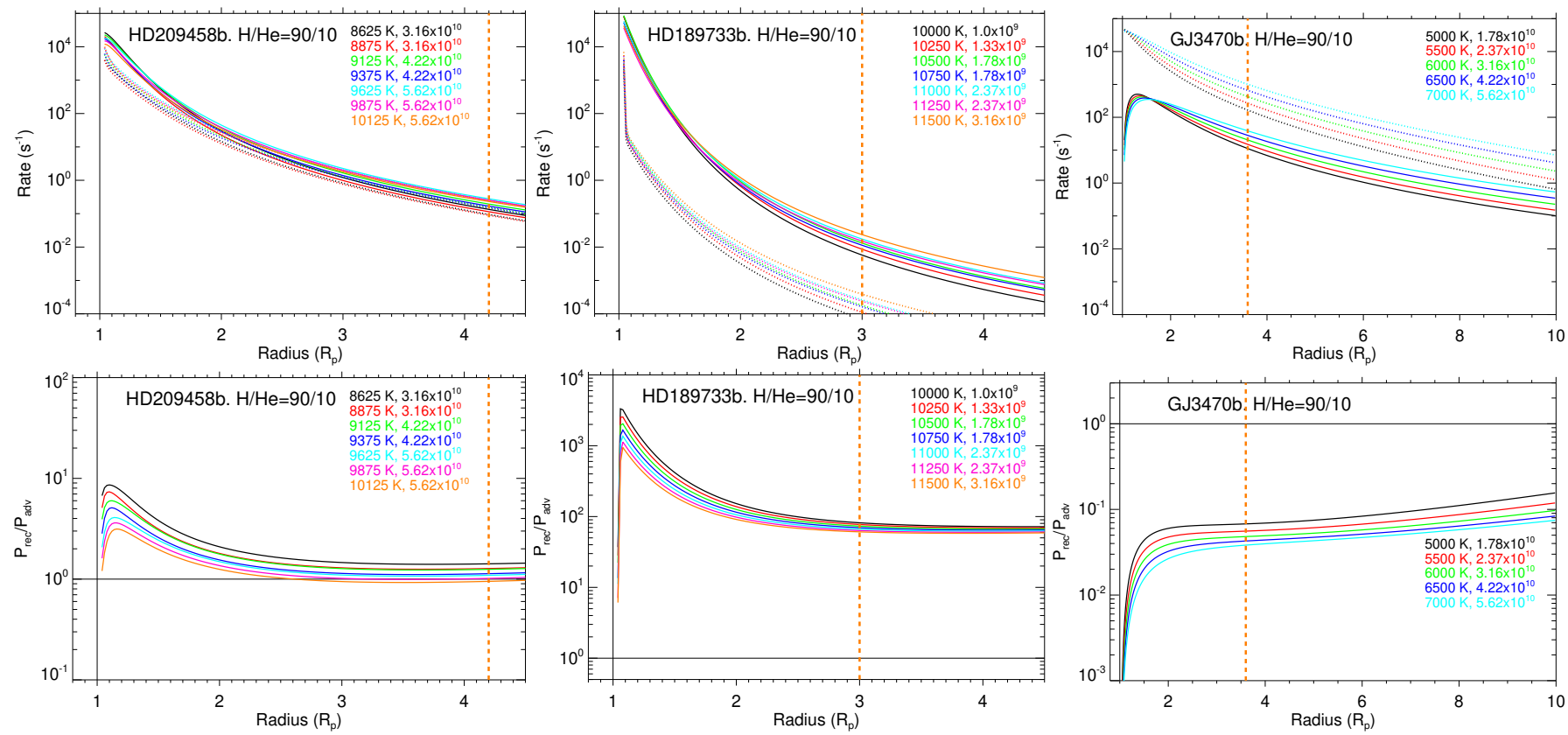

Fig. B.2. Same as Fig. 3 but for an H/He ratio of 90/10 (see Fig. 5 in Lampón et al. 2021). Upper row: rates of H by recombination (solid lines) and advection (dotted lines). Lower row: ratios of recombination to advection. The vertical dashed orange lines indicate the mean Roche lobes. We note the different scales of the $x$ and $y$ axes. 
M. Lampón et al.: Evidence of energy-, recombination-, and photon-limited escape regimes

Table B.1. System parameters.

\begin{tabular}{lccc}
\hline \hline System & HD 209458 & HD 189733 & GJ 3470 \\
\hline$M_{\star}\left(M_{\odot}\right)^{(a)}$ & $1.119_{-0.033}^{+0.033}$ & $0.846_{-0.049}^{+0.06}$ & $0.476_{-0.019}^{+0.019}$ \\
$a(\mathrm{au})^{(b)}$ & $0.04707_{-0.00046}^{+0.00046}$ & $0.0332_{-0.001}^{+0.001}$ & $0.0348_{-0.0014}^{+0.0014}$ \\
$M_{\mathrm{P}}\left(M_{\text {Jup }}\right)^{(c)}$ & $0.685_{-0.014}^{+0.015}$ & $1.162_{-0.039}^{+0.058}$ & $0.036_{-0.002}^{+0.002}$ \\
$R_{\mathrm{P}}\left(R_{\text {Jup }}\right)^{(c)}$ & $1.359_{-0.019}^{+0.016}$ & $1.23_{-0.03}^{+0.03}$ & $0.360_{-0.01}^{+0.01}$ \\
$\Phi\left(\Phi_{\text {Jup }}\right)^{(d)}$ & 0.504 & 0.944 & 0.100 \\
$R_{\text {lobe }}\left(R_{\mathrm{P}}\right)^{(e)}$ & 4.2 & 3.0 & 3.6 \\
$F_{\text {XUV }}(f)$ & 2.358 & 56.740 & 3.928 \\
\hline
\end{tabular}

Notes. ${ }^{(a)}$ Stellar masses of HD 209458, HD 189733, and GJ 3470 from Torres et al. (2008), de Kok et al. (2013), and Pallé et al. (2020), respectively. ${ }^{(b)}$ Orbital separation from Torres et al. (2008), Agol et al. (2010), and Bonfils et al. (2012). ${ }^{(c)}$ Planetary mass and radius of HD 209458 b from Torres et al. (2008); of HD 189733b from de Kok et al. (2013) and Baluev et al. (2015); and of GJ 3470 b from Pallé et al. (2020). ${ }^{(d)}$ Gravitational potential. ${ }^{(e)}$ Roche lobes of HD 209458 b, HD 189733 b, and GJ 3470 b by Salz et al. (2016a), Eggleton (1983), and Bourrier et al. (2018), respectively. ${ }^{(f)}$ XUV flux in units of $10^{3} \mathrm{erg} \mathrm{cm}^{-2} \mathrm{~s}^{-1}$ at $\lambda<912 \AA$ at the planetary separation calculated by Lampón et al. (2020) for HD 209458 b and by Lampón et al. (2021) for HD $189733 \mathrm{~b}$ and GJ $3470 \mathrm{~b}$.

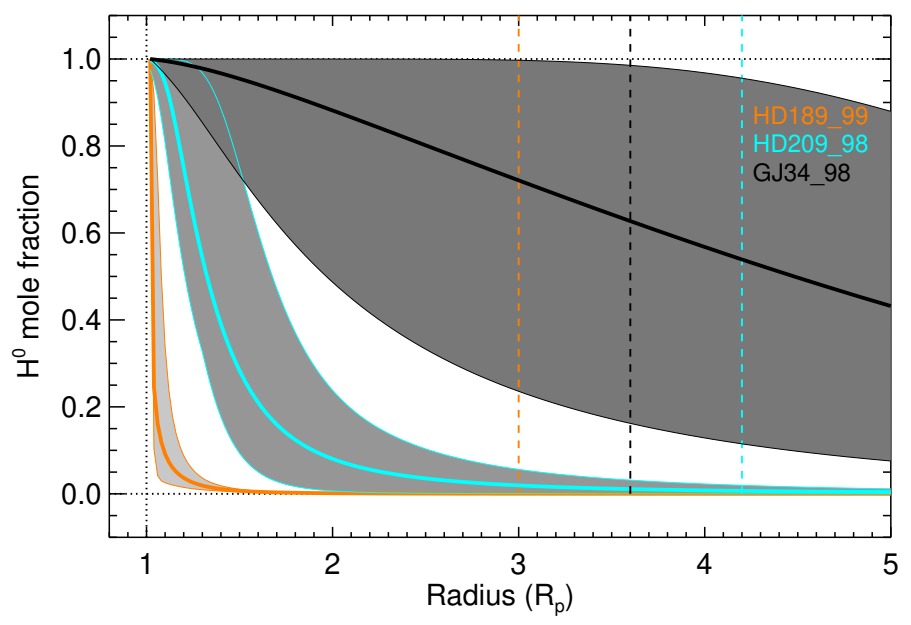

Fig. B.3. Same as Fig. 2 but for the extended $T-\dot{M}$ ranges (see the dotted lines in Fig. 1). The thicker solid lines are the mean profiles. The vertical dashed lines are the mean Roche lobes.

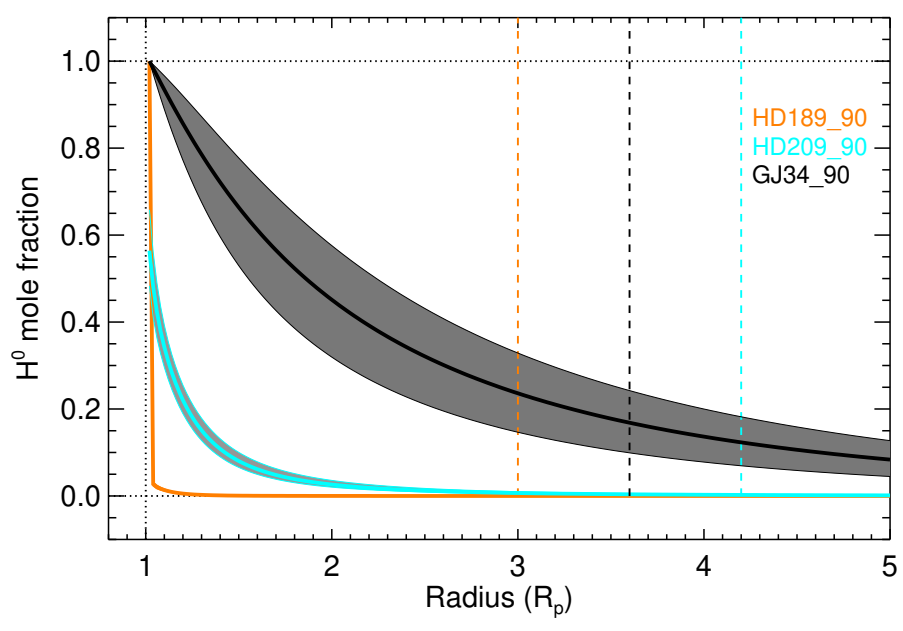

Fig. B.4. Same as Fig. 2 but for an H/He mole fraction of 90/10 (see Fig. 5 in Lampón et al. 2021). The thicker solid lines are the mean profiles. The vertical dashed lines are the mean Roche lobes. 